\title{
Effect of Reaction Time and Stability Properties of Gold Nanoparticles Synthesized by $p$-Aminobenzoic Acid and $p$-Aminosalicylic Acid
}

\author{
Abdul Aji, Sri Juari Santosa, and Eko Sri Kunarti* \\ Department of Chemistry, Faculty of Mathematics and Natural Sciences, Universitas Gadjah Mada, \\ Sekip Utara, Yogyakarta 55281, Indonesia
}

${ }^{*}$ Corresponding author:

email:eko_kunarti@ugm.ac.id

Received: April 2, 2019

Accepted: August 14, 2019

DOI: $10.22146 /$ ijc. 44674

\begin{abstract}
In this work, we determined the influence of reaction time in the synthesis of gold nanoparticles (AuNPs) by $\mathrm{p}$-aminosalicylic acid and $\mathrm{p}$-aminobenzoic acid as reducing agents. Besides working as a reducing agent, the p-aminobenzoic acid and paminosalicylic acid also simultaneously played a role as a capping agent/stabilizing agent. Gold ions were first mixed with the $\mathrm{pH}$ adjusted $\mathrm{p}$-aminobenzoic acid and $\mathrm{p}$ aminosalicylic acid. The mixture was then heated in boiling water at $86{ }^{\circ} \mathrm{C}$. The formation of AuNPs was indicated by the appearance of red color and analyzed with $U V / V i s$ spectrophotometry to evaluate their surface plasmon resonance (SPR) absorption in the wavelength range of 400-800 $\mathrm{nm}$. The reducing ability of the reducing agents was affected by its structure. Gold nanoparticles that were synthesized with p-aminosalicylic acid were more stable, formed faster and had a smaller size than its counterpart that were synthesized with p-aminobenzoic acid. The stability test over a period of 5 months showed that the AuNPs were relatively stable.
\end{abstract}

Keywords: gold nanoparticles; p-aminosalicylic acid; p-aminobenzoic acid

\section{- INTRODUCTION}

Metal nanoparticles have become an incessant part of the current research in nanotechnology. They exhibit unusual physical and chemical properties which are quite different from their bulk counterparts [1]. When particles are reduced to their nano level, their physical and chemical properties (e.g., melting point, fluorescence, electrical conductivity, magnetic permeability, and chemical reactivity) can drastically change. The changes in these properties are highly influenced by their size, shape, and nature of the surrounding environment [2]. Application of nanomaterial follows its unique properties including high surface to volume ratio, high surface energy, and unique mechanical, thermal, electrical, magnetic and optical behaviors [3].

Gold nanoparticles are the most compatible nanomaterial for preparation of engineered nanoplatforms in smart sensing devices. Surface plasmon resonance property of gold nanoparticles makes them most suitable engineered nanomaterial for bioimaging, biomedical therapeutics, and biodiagnostic tools. Gold nanoparticles colloids have attracted increasing attention due to their unique properties in multi-disciplinary research fields [4].

A gold nanoparticle is generally synthesized through the reduction of the $\mathrm{HAuCl}_{4}$ solution with $\mathrm{NaBH}_{4}$ in high temperature [5]. However, the synthesized AuNPs tend to aggregate as soon as it is formed. The use of other reducing agents, such as citric acid, polyols, sugars, hydrazine, formaldehyde, hydrogen peroxide, and ascorbic acid still could not solve the aggregation problem of gold nanoparticles. To protect the particles from aggregation and reduce the collision possibility between particles of gold, in order to make AuNPs become stable, the researchers use stabilizers [6]. The most common capping agents used in previous studies were SDS [7], PVA [8], cysteamine [9], and surfactant [10]. The stabilizing agent was added after the reduction process to maintain the size of gold nanoparticles. The formation of AuNPs are indicated by the colloid formation with red or purple color and has strong adsorption of visible light at the wavelengths between 520-550 nm [11]. Nguyen et al. synthesized AuNPs by capping $\mathrm{Au}^{3+}$ ion using a CTAB 
and then reducing with $\mathrm{NaBH}_{4}[12] . \mathrm{NaBH}_{4}$ was also used as a reducing agent for gold nanoparticles. Wu et al. [13] added trisodium citrate as a stabilizing agent and used it for pesticides detection. However, the use of two different compounds was less effective.

Previous studies have been conducted by using the same chemicals as reducing agent and stabilizer. In 2004, Aslam et al. synthesized gold nanoparticles using oleylamine as both a reducing agent and stabilizing agent. The particle size of the gold nanoparticles that were synthesized with this method was 8-12 nm [14]. In 2016, Gusrizal et al. had synthesized AgNPs using p-hydroxy benzoic acid and $m$-hydroxybenzoic acid as both a reducing agent and capping agent [15]. The reduction process occurs by the presence of hydroxyl group $(-\mathrm{OH})$ and the amino group that can reduce $\mathrm{Au}^{3+}$ to $\mathrm{Au}^{0}$ and the presence of the carboxylic group $(-\mathrm{COOH})$ that can facilitate electrostatic interactions with $\mathrm{Au}^{3+}$ ions in solution. This interaction can prevent the aggregation of $\mathrm{Au}^{3+}$ ions so it will not precipitate.

In previous work, we reported the optimalization condition of gold nanoparticles that were synthesized with the double function of $p$-aminobenzoic acid and $p$ aminosalicylic acid as reducing and capping agents in the formation of AuNPs. Several parameters, such as $\mathrm{pH}$, the concentration of reducing agent and precursor concentration have been reported [16]. In this work, we studied the effect of reaction time in the synthesis of gold nanoparticles using $p$-aminobenzoic acid and $p$ aminosalicylic acid as a reducing agent and stabilizing agent. In addition, we reported the advanced study of the stability properties of gold nanoparticles that were formed. Effect of reaction time in the formation of gold nanoparticles was studied using UV-visible spectroscopy to monitor the appearance of surface plasmon resonance absorption. Stability properties of the gold nanoparticles were determined by UV-visible spectroscopy, transmission electron microscopy (TEM), zeta potential data and dynamic light scattering (DLS).

\section{- EXPERIMENTAL SECTION}

\section{Materials}

The materials that were used in this research were gold(III) chloride acid solution $\left(\mathrm{HAuCl}_{4} 100 \mathrm{ppm}\right)$, p-aminobenzoic acid $\left(\mathrm{C}_{7} \mathrm{H}_{7} \mathrm{NO}_{2}\right.$, Merck), p-aminosalicylic acid $\left(\mathrm{C}_{7} \mathrm{H}_{7} \mathrm{NO}_{3}\right.$, Merck), and sodium hydroxide $(\mathrm{NaOH}$, Merck). For the preparation of the mixture solution, double distilled water was used in all experiments.

\section{Instrumentation}

SPR spectra of gold nanoparticles were obtained by measurement using a UV-visible spectrophotometer (Shimadzu UV-1700 PharmaSpec instrument). Transmission electron microscope (JEOL JEM-1400) was used to determine the size and morphology of gold nanoparticles. Fourier transmission infrared spectrophotometer (Shimadzu FTIR Prestige-21) was used to identify functional groups present in the gold nanoparticles and to investigate the interaction of the AuNPs surface with the reducing agents. Zeta potential data was determined using Horiba SZ-100.

\section{Procedure}

\section{Sample preparation}

AuNPs were synthesized by reducing $\mathrm{HAuCl}_{4}$ solution with $p$-aminobenzoic acid and $p$-aminosalicylic acid as reducing agents at $\mathrm{pH} 12$ and 13, respectively, by adding a solution of $\mathrm{NaOH}$. Ten $\mathrm{mL}$ of $20 \mathrm{mM}$ reducing agents were put into a beaker glass and added with $10 \mathrm{~mL}$ of $100 \mathrm{ppm} \mathrm{HAuCl}_{4}$ solution. The mixture was stirred vigorously and heated at temperature of $86{ }^{\circ} \mathrm{C}$. The reaction time was varied from $0-100 \mathrm{~min}$ to obtain the optimum reaction time. Formation of gold nanoparticles was visually identified by observing the color change of the reaction from light yellow to pink or light red. All solutions were also monitored by UV/Vis Spectrophotometer at a wavelength of $300-800 \mathrm{~nm}$. The presence of maximum absorption at the wavelength range of $520-550 \mathrm{~nm}$ showed that AuNPs are formed [16].

\section{Characterization of gold nanoparticles}

The UV-visible spectra of the samples were recorded in the wavelength range of $300-800 \mathrm{~nm}$ at room temperature. All UV-Visible measurements were performed using $1 \mathrm{~cm}$ optical path length quartz cuvette. To obtain the stability of gold nanoparticles, UV-visible measurements were performed periodically for a period of 5 months. 
The solid sample for FTIR analysis was prepared using gold nanoparticles recovered from colloidal via centrifugation at $12000 \mathrm{rpm}$ and then were dried at $60^{\circ} \mathrm{C}$ in an oven. Approximately, a sample of $5 \mathrm{mg}$ was mixed with $\mathrm{KBr}$ and condensed into pellet using a hydraulic press. This method was used for all FTIR spectra analysis.

The sample for TEM analysis was prepared by dropping the samples and depositing them in TEM carbon covered copper grids, and letting it evaporate at room temperature. The TEM image was recorded with a $120 \mathrm{kV}$ acceleration voltage. The size of the particles was calculated using ImageJ software.

\section{- RESULTS AND DISCUSSION}

\section{Synthesis of Gold Nanoparticles}

Several synthesis parameters of gold nanoparticles using $p$-aminobenzoic acid and $p$-aminosalicylic acids, such as $\mathrm{pH}$ condition, precursor concentration, and reducing agent concentration were reported in previous work [16]. Based on previous work, in this study, the formation of gold nanoparticles was performed by mixing $\mathrm{HAuCl}_{4}$ solution with $p$-aminobenzoic acid and $p$-aminosalicylic acid as reducing agents at $\mathrm{pH} 12$ and 13, respectively, by adding a solution of $\mathrm{NaOH}$. When $p$-aminobenzoic acid and $p$-aminosalicylic acid reduced the gold ions, the color of the solution changed to pink or red color indicating the formation of gold nanoparticles. The change in color of the reaction medium indicated the formation of gold nanoparticles due to surface plasmon resonance phenomenon. To prove the formation of gold nanoparticles, the solutions were analyzed by UV-Vis spectrophotometer at the wavelength range of $300-800 \mathrm{~nm}$. The formation of gold nanoparticles was indicated by the maximum absorbance which happened in the wavelength range of 520-550 nm [17]. The result in Fig. 1 showed that the gold nanoparticles were formed. The narrow SPR peak of the gold nanoparticles that were synthesized by $p$-aminosalicylic acid indicated that the size of the AuNPs were uniform. On the other hand, the broadening SPR peak of the gold nanoparticles that were synthesized by $p$-aminobenzoic acid indicated that the size of the AuNPs were non-uniform. The SPR peak showed only one plasmon band centered at about $520 \mathrm{~nm}$, indicating that

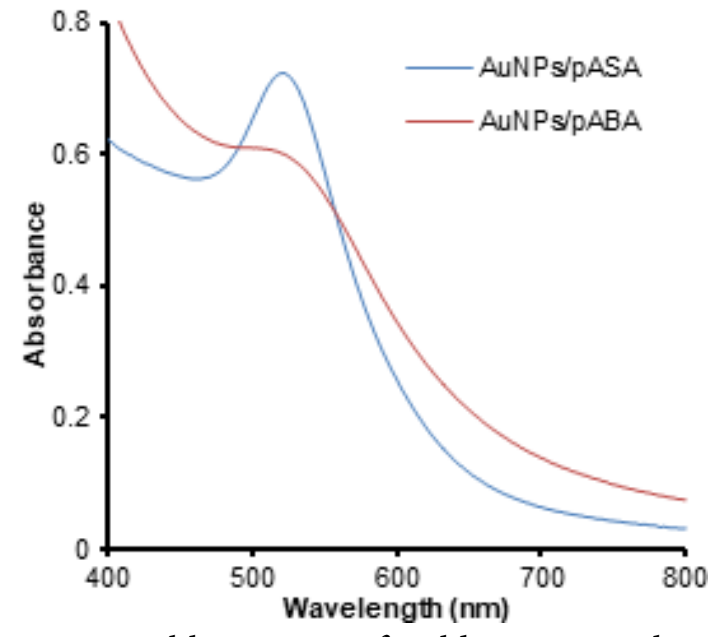

Fig 1. UV-visible spectra of gold nanoparticles made from $100 \mathrm{ppm} \mathrm{HAuCl}_{4}$ and $20 \mathrm{mM}$ reducing agent

the shape of the AuNPs were spherical with the size of 2-50 nm. Meanwhile, two SPR bands usually appear when the symmetry is reduced from spherical to cylindrical, i.e., in gold nanorods [18]. Fig. 1 showed that $p$-aminosalicylic acid as a reducing agent produced more gold nanoparticles than $p$-aminobenzoic acid on equal synthesis conditions. This data indicated that the structure of the reducing agents influenced the reducing ability.

\section{Effect of Reaction Time on the Formation of Gold Nanoparticles}

Another factor that is very important in the synthesis of gold nanoparticles using $p$-aminobenzoic acid and $p$-aminosalicylic acid is reaction time. Kumari et al. demonstrated in their study the evolution of the morphology of gold nanoparticles from nanospheres to triangular nanoprisms with an increase in time in $T$. asperellum [19]. The difference in reaction time using the same method could induce different sizes of gold nanoparticles that could be synthesized. The spherical nanoparticles can be produced in a relatively short reaction time. The reduction of $\mathrm{Au}^{3+}$ ions to $\mathrm{Au}^{0}$ mediated by $p$-aminobenzoic acid and $p$-aminosalicylic acid at optimum conditions was examined spectroscopically at different reaction times and the stacked spectra are displayed in Fig. 2. All reactions showed the formation of gold nanoparticles. Although the intensity of peaks was different at different reaction times, all peaks were centered at $520-530 \mathrm{~nm}$. 

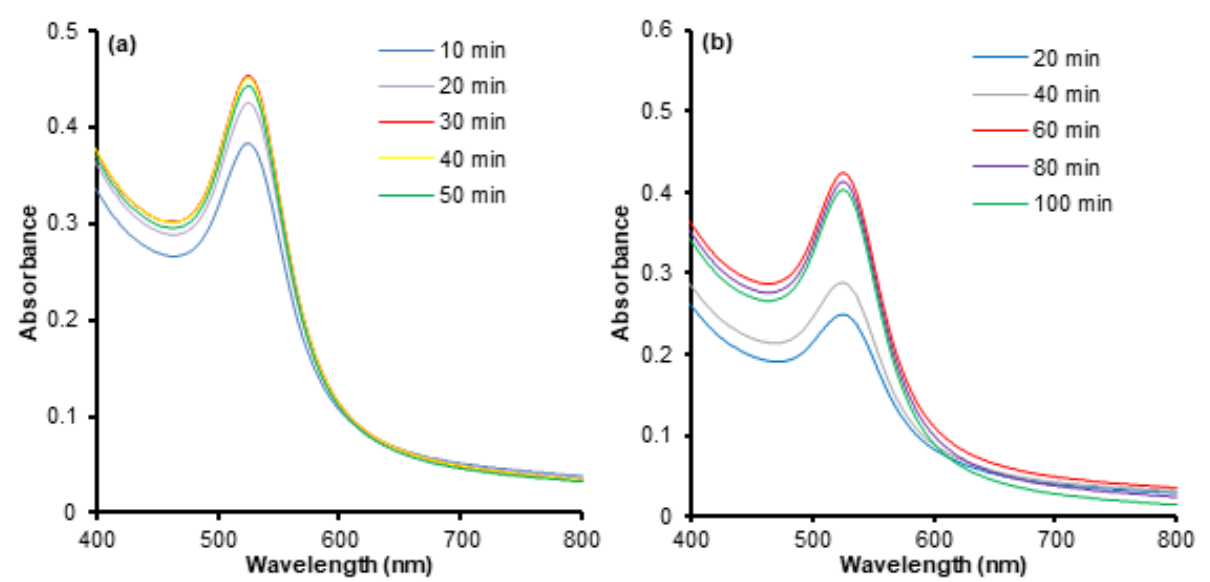

Fig 2. UV/Vis spectra of gold nanoparticles with various reaction times with (a) $p$-aminosalicylic acid at $\mathrm{pH} 12$ and (b) p-aminobenzoic acid at $\mathrm{pH} 13$

Fig. 2 shows that the formation of gold nanoparticles with $p$-aminosalicylic acid as the reducing agent was faster than with $p$-aminobenzoic acid. Using $20 \mathrm{mM} p$-amino

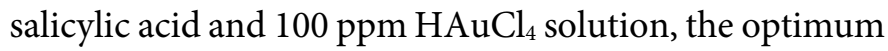
reaction time was $30 \mathrm{~min}$. Increase in absorbance during $10 \mathrm{~min}$ to $30 \mathrm{~min}$ of reaction time was attributed to the reduction of gold precursors and an increase in the population of AuNPs. The absorbance value became constant and slightly decreased after 30 min of reaction time due to the complete reduction of available gold ions. The synthesis was found to be completed within $30 \mathrm{~min}$ because no further rise in the intensity of the SPR band was detected. In addition, the optimum reaction time using $20 \mathrm{mM}$-aminobenzoic acid and $100 \mathrm{ppm} \mathrm{HAuCl}_{4}$ solution took $60 \mathrm{~min}$. As the reaction continues, the intensity of this band increased together with a little blue shift attributed to the creation of small sized spherical nanoparticles. The synthesis was found to be completed within $60 \mathrm{~min}$ and no further rise in the intensity of the SPR band was detected.

After the reaction, the colloidal gold nanoparticles was stored for 7 days and its absorbance was re-measured. This is done to evaluate the influence of heating time on the stabilization performance of AuNPs that was synthesized by $p$-aminobenzoic acid and $p$-aminosalicylic acid. Based on the data in Fig. 3, it can be explained that the gold nanoparticles that were formed by $p$-amino salicylic acid at less than 30 min of heating time were not stable because its absorbance still increased after 7 days. This data showed that the formation of gold nanoparticles still continued when the reaction was stopped. The gold nanoparticles formed by $p$-aminosalicylic acid at $30 \mathrm{~min}$
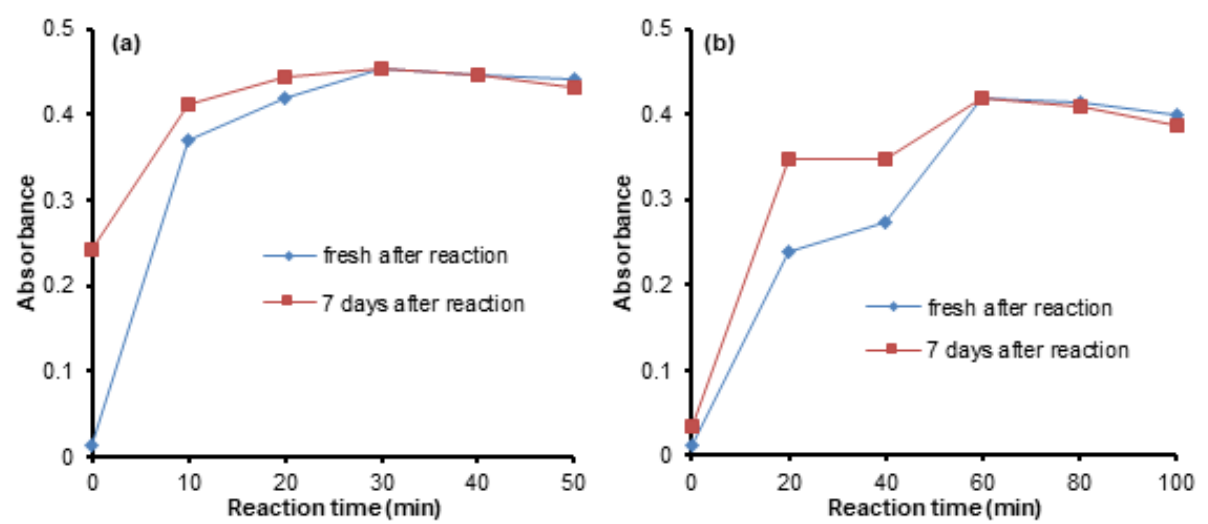

Fig 3. UV/Vis absorbance of fresh-synthesized and 7 days-stored of AuNPs with various reaction times with (a) $p$ aminosalicylic acid at pH 12 and (b) p-aminobenzoic acid at pH 13 
reaction time was most stable, shown by the unchanged value of absorbance after 7 days. Meanwhile, the gold nanoparticles formed by $p$-aminosalicylic acid at more than $30 \mathrm{~min}$ reaction time was less stable because its absorbance slightly decreased after 7 days. The gold nanoparticles formed by $p$-aminobenzoic acid at $60 \mathrm{~min}$ reaction time was most stable shown by the unchanged value of absorbance after 7 days. After 7 days stored, the gold nanoparticles formed by $p$-aminobenzoic acid had the same pattern with the gold nanoparticles formed by $p$-aminosalicylic acid. The gold nanoparticles that were formed by $p$-aminobenzoic acid at less than $60 \mathrm{~min}$ reaction time was not stable because its absorbance still increased after 7 days. On the other hand, the gold nanoparticles formed by $p$-aminobenzoic acid at more than 60 min reaction time was less stable because its absorbance slightly decreased after 7 days. It is predicted that the longer the solution is heated, the molecules in the solution would still be active, even when the heating process has stopped. The active molecules will react further so the reduction process would still occur even when the heating has been stopped [20].

The best reducing and stabilizing performance of $p$-aminosalicylic was obtained at the reaction time of $30 \mathrm{~min}$. This optimum reaction time was faster than using $p$-aminobenzoic acid as a reducing and stabilizing agent, which needed $60 \mathrm{~min}$ to reach the optimum reaction time. In $p$-aminosalicylic, the hydrogen atom at the hydroxyl group and the oxygen atom at the carboxyl group could produce intramolecular hydrogen bonding. Intramolecular hydrogen bonding in the $p$-aminosalicylic acid structure

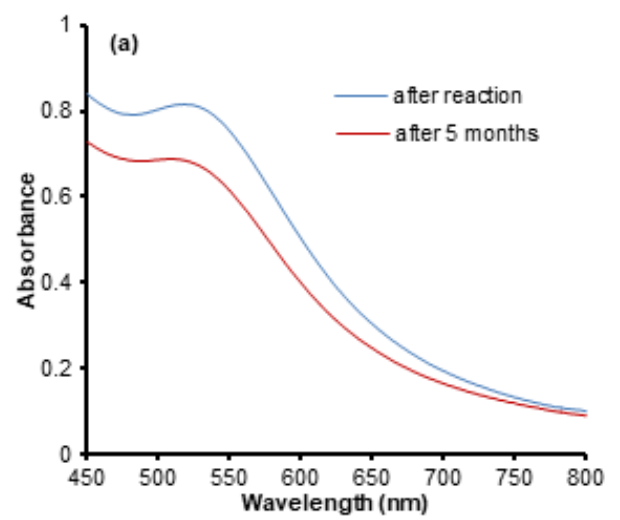

causes the reduction reaction to be more effective [21]. Therefore, the performance of $p$-aminosalicylic acid as a reducing agent and capping agent was better than $p$-aminobenzoic acid.

\section{p-Aminobenzoic Acid and p-Aminosalicylic Acid as a Capping Agent}

The stability test was performed to gold nanoparticles produced by the reaction of $100 \mathrm{ppm}$ $\mathrm{HAuCl}_{4}$ solution with $20 \mathrm{mM}$ reducing agent to describe the role of $p$-aminobenzoic acid and $p$-aminosalicylic acid as a capping agent. The gold nanoparticles were stored in a capped bottle at room temperature condition for 5 months. After 5 months of observation, all gold nanoparticles that was resulted did not give significant decrease in absorbance value (Fig. 4), only 0.126 (15.47\%) for $p$-aminosalicylic acid and 0.210 (29.13\%) for $p$-aminobenzoic acid. This data described that all resulted gold nanoparticles were relatively stable until 5 months.

Other than the absorbance decrease, the stability of gold nanoparticles can also be studied from the maximum wavelength shift. The maximum wavelength shift happened after 5 months as far as $3 \mathrm{~nm}$ for $p$-aminosalicylic acid and $6.5 \mathrm{~nm}$ for $p$-aminobenzoic acid. The longer storage will decrease the gold nanoparticles stability because the particles are suspended by agglomeration (Fig. 5). However, after the shift, the maximum wavelength was still located around $520 \mathrm{~nm}$. It means that the gold nanoparticles were relatively stable over a period of 5 months.

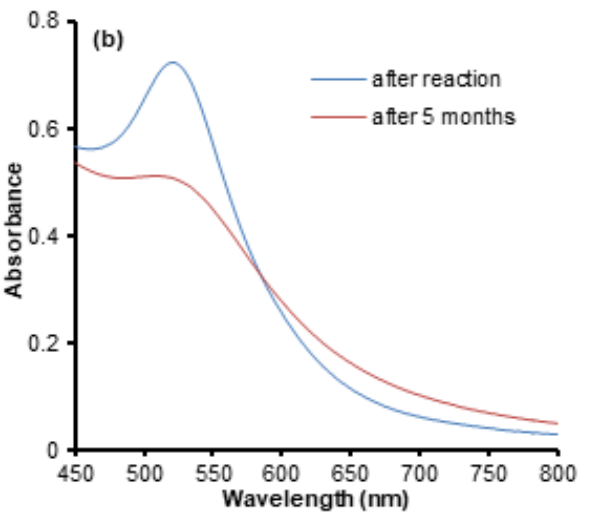

Fig 4. UV/Vis spectra of fresh and after 5 months stored of AuNPs at room temperature (a) AuNPs produced from $p$ aminosalicylic acid (b) AuNPs produced from $p$-aminobenzoic acid 

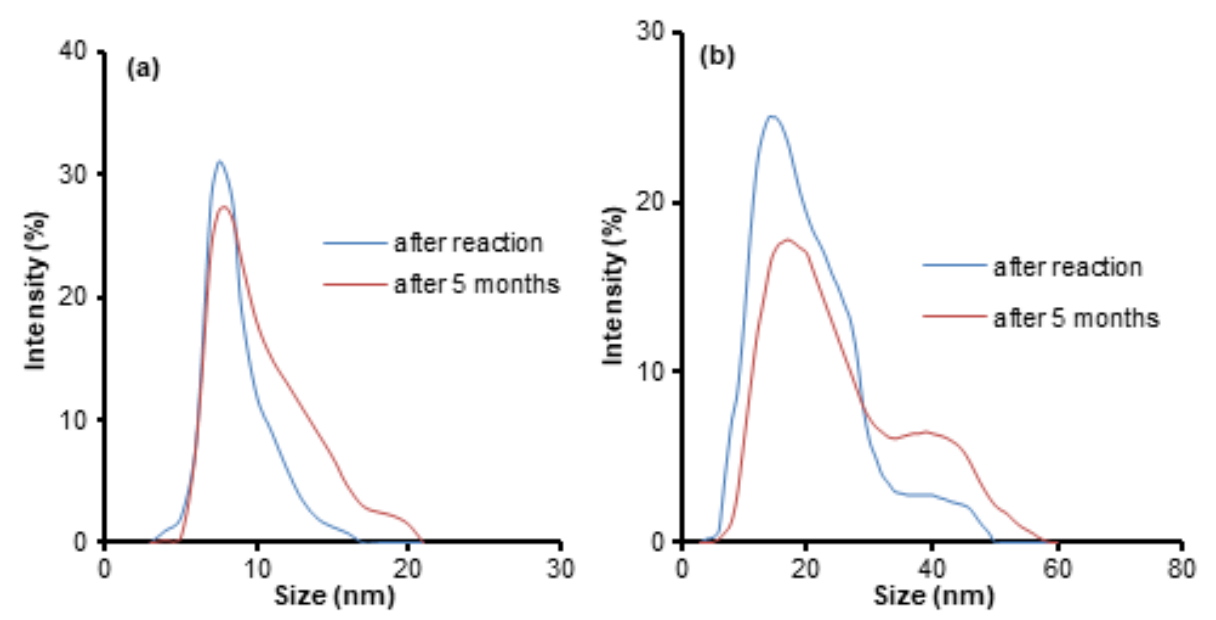

Fig 5. DLS spectra of fresh and after 5 months stored of AuNPs at room temperature (a) AuNPs produced from $p$ aminosalicylic acid (b) AuNPs produced from $p$-aminobenzoic acid

For comparison, the gold nanoparticles that were synthesized by $p$-aminosalicylic acid was more stable than the gold nanoparticles that resulted from $p$-aminobenzoic acid. This fact may indicate that the existence of the hydroxyl group in $p$-aminosalicylic acid can increase the stability of gold nanoparticles. In $p$-aminosalicylic, the hydrogen atom at the hydroxyl group and the oxygen atom at the carboxyl group could produce intramolecular hydrogen bonding. Intramolecular hydrogen bonding in the $p$-aminosalicylic acid structure can cause the reduction reaction to be more effective and increase the stability of the AuNPs.

The size and morphology of the resulted gold nanoparticles were observed using DLS data and TEM, shown in Fig. 6. The TEM results showed that both reducing agents gave the same shape but different size of gold nanoparticles. The $p$-aminosalicylic acid reducing agent (Fig. 5(a)) gave a round shape and smaller particle size than gold nanoparticles that were reduced by $p$-aminobenzoic acid. The $p$-aminobenzoic acid reducing agent (Fig. 6(b)) gave a round shape and less uniform size of gold nanoparticles. The aggregation did not happen with both $p$-aminobenzoic acid and $p$-aminosalicylic acid reducing agent, which showed that the reducing agents can also act as stabilizers.

The stability of the colloidal gold nanoparticles is often described by their zeta potential value [22]. The higher zeta potential value indicates the smaller size and higher stability of gold nanoparticles. In this work, gold nanoparticles that were synthesized by $p$-aminosalicylic acid had a higher zeta potential value than gold nanoparticles that were synthesized by $p$-aminobenzoic acid. The zeta potential value of the AuNPs that were synthesized by $p$-aminosalicylic acid and by p-aminobenzoic acid was $-42.8 \mathrm{mV}$ and $-32.4 \mathrm{mV}$, respectively. Particles with zeta potentials of more than $+30 \mathrm{mV}$ and more negative than $-30 \mathrm{mV}$ are normally considered stable. The greater the zeta potential value causes higher repulsion between the particles, so aggregation could be prevented [23]. Therefore, the size of gold nanoparticles that were synthesized by $p$-aminosalicylic acid was smaller and more stable than the ones synthesized by $p$-aminobenzoic acid. The negative value in this data indicates the presence of negative charge at the surface of the gold nanoparticles [24]. This zeta potential value is in agreement with the size data from the TEM image and the stability data that was determined from the UV/Vis spectra of the gold nanoparticles.

The presence of $p$-aminosalicylic acid and $p$ aminobenzoic acid as a capping agent should be a result from the interaction of gold nanoparticles with the functional groups of the reducing agent. FTIR analysis was performed to characterize any chemical changes that occurred during the synthesis of gold nanoparticles with $p$-aminosalicylic acid and $p$-aminobenzoic acid as a 

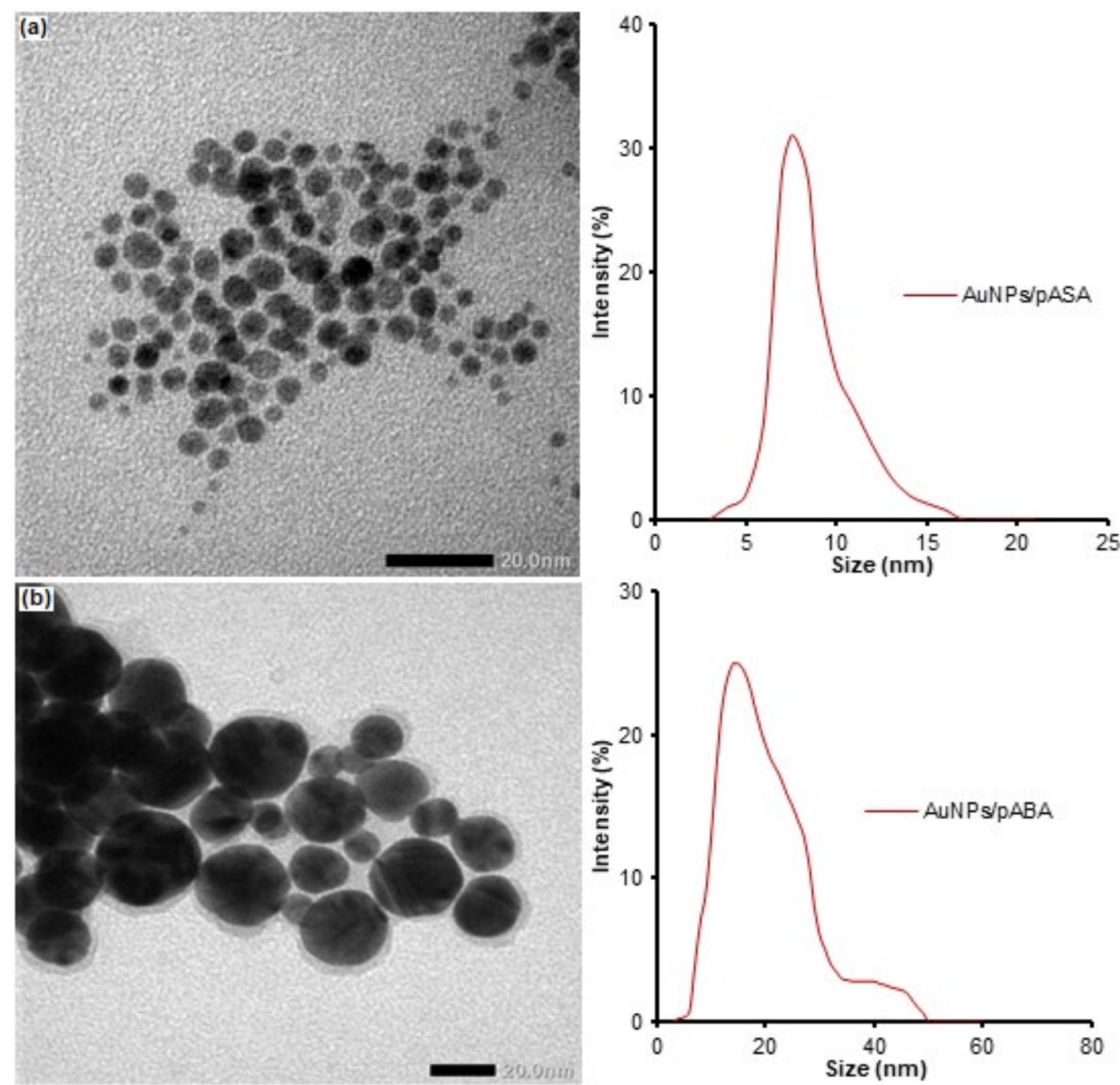

Fig 6. The TEM image and particle size distribution of gold nanoparticles synthesized by a reducing agent (a) $p$ aminosalicylic acid at $\mathrm{pH} 12$ (b) $p$-aminobenzoic acid at $\mathrm{pH} 13$

capping agent (Fig. 7). Because no additional capping agent was used in this experiment, it is clear that the reducing agents not only acted as a reducing agent but also as a capping agent.

FTIR spectra of p-aminobenzoic acid showed two strong absorption bands at 3476 and $3383 \mathrm{~cm}^{-1}$, while p-aminosalicylic acid showed two strong absorption bands at 3490 and $3381 \mathrm{~cm}^{-1}$ which are due to the asymmetric and symmetric $\mathrm{N}-\mathrm{H}$ stretching vibrations of the $-\mathrm{NH}_{2}$ group. The band of valence vibrations of the $\mathrm{C}=\mathrm{O}$ group in the carboxyl acid was observed at $1638 \mathrm{~cm}^{-1}$ for $p$-aminobenzoic acid and $1609 \mathrm{~cm}^{-1}$ for $p$-aminosalicylic acid. The absorption bands at 1604, 1575 and $1524 \mathrm{~cm}^{-1}$ correspond to the valence vibrations of
$\mathrm{C}=\mathrm{C}$ bonds in the benzene ring. The absorption band of the valence vibrations of the $\mathrm{C}-\mathrm{N}$ bond in the amino group connected with the benzene ring was observed at $1314 \mathrm{~cm}^{-1}$ for $p$-aminobenzoic acid and $1317 \mathrm{~cm}^{-1}$ for $p$-aminosalicylic acid. It can be observed that the characteristic spectral bands of $p$-aminobenzoic acid and $p$-aminosalicylic were completely shifted or changed by the functionalization of the AuNPs. Additionally, the characteristic features of stretching modes of benzene were observed at 1597, 1512 and $1442 \mathrm{~cm}^{-1}$ and $-\mathrm{COOH}$ and $-\mathrm{NH}_{2}$ stretching modes were observed at 1635 and $3425 \mathrm{~cm}^{-1}$, respectively. The results indicate that $p$-aminobenzoic acid and $p$-aminosalicylic molecules were successfully assembled on the surfaces of the AuNPs. 


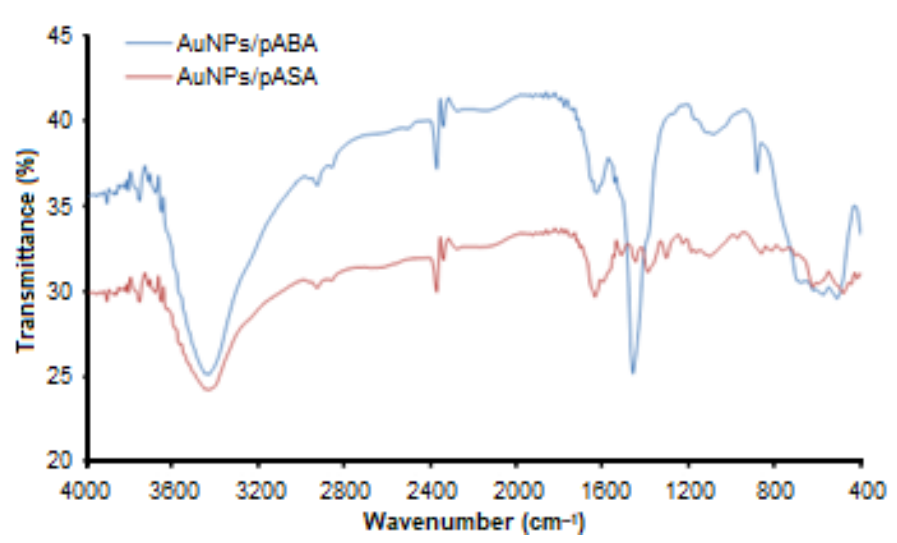

Fig 7. FTIR spectra of gold nanoparticle that synthesized by $p$-aminosalicylic acid at $\mathrm{pH} 12$ (AuNPs/pASA) and $p$-aminobenzoic acid at $\mathrm{pH} 13$ (AuNPs/pABA)

Furthermore, the spectral changes give clear evidence of amine groups interactions on the surfaces of the AuNPs, which was well agreed with the reported methods on the functionalization of metal NPs with two organic molecules including amino group containing ligand [25].

\section{- CONCLUSION}

$p$-Aminosalicylic acid and $p$-aminobenzoic acid have been successfully used as reducing agents and also stabilizing agents in the synthesis process of gold nanoparticles. This reaction process was highly influenced by reaction time. AuNPs that were synthesized by $p$-aminosalicylic acid was produced faster than the AuNPs that were synthesized by $p$-aminobenzoic acid. The stability of the AuNPs that were synthesized by $p$-aminosalicylic acid was better than the AuNPs that were synthesized by $p$-aminobenzoic acid until 5 months of observation. In addition, the $p$-aminosalicylic acid reducing agent can produce smaller gold nanoparticles than the ones by $p$-aminobenzoic acid. Results from UV/vis spectra, TEM, Zeta potential and FTIR proved that $p$-aminosalicylic acid as a reducing agent and also a stabilizing agent had better performance than $p$-aminobenzoic acid.

\section{- ACKNOWLEDGMENTS}

The authors give thanks to the Ministry of Research Technology and Higher Education of the Indonesian Republic which has partly supported this research activity by providing a scholarship of Master Education Program
Leading to Doctoral Degree for Excellent Graduates (PMDSU).

\section{- REFERENCES}

[1] Tyagi, H., Kushwaha, A., Kumar, A., and Aslam, M., 2011, pH Dependent synthesis of stabilized of gold nanoparticles using ascorbic acid, Int. J. Nanosci., 10 (4-5), 857-860.

[2] Shah, M., Badwaik, V., Kherde, Y., Waghwani, H.K., Modi, T., Aguilar, Z.P., Rodgers, H., Hamilton, W., Marutharaj, T., Webb, C., Lawrenz, M.B., and Dakshinamurthy, R., 2014, Gold nanoparticles: Various methods of synthesis and antibacterial activity, Front. Biosci., 19, 1320-1344.

[3] Chen, G., Roy, I., Yang, C., and Prasad, P.N., 2016, Nanochemistry and nanomedicine for nanoparticlebased diagnostics and therapy, Chem. Rev., 116 (5), 2826-2885.

[4] Verma, N.H., Singh, P., and Chavan, R.M., 2014, Gold nanoparticle: Synthesis and characterization, Vet. World, 7, 72-77.

[5] Turkevich, J., Stevenson, P.C., and Hillier, J., 1951, A study of the nucleation and growth process in the synthesis of colloidal gold, Discuss. Faraday Soc., 11, 55-75.

[6] Bin Ahmad, M., Lim, J.J., Shameli, K., Ibrahim, N.A., and Tay, M.Y., 2011, Synthesis of silver nanoparticles in chitosan, gelatin and chitosan/gelatin bionano composites by a chemical reducing agent and their characterization, Molecules, 16 (9), 7237-7248.

[7] Barman, G., Maiti, G., and Laha, J.K., 2013, Biofabrication of gold nanoparticles using aqueous extract of red tomato and its use as a colorimetric sensor, Nanoscale Res. Lett., 8 (1), 181-190.

[8] Walekar, L.S., Pawar, S.P., Gore, A.H., Suryavanshi, V.D., Undare, S.S., Anbhule, P.V., Patil, S.R., and Kolekar, G.B., 2016, Surfactant stabilized AgNPs as a colorimetric probe for simple and selective detection of hypochlorite anion in aqueous solution: Environmental sample analysis, Colloids Surf., A, 491, 78-85.

[9] Choi, H., Kang, T., Um, K., and Lee, K., 2014, Reduction of silver ions in gold nanoparticle 
suspension on detection of dihydroxybenzene isomers, Colloids Surf., 459, 120-127.

[10] Zhao, P., Li, N., and Astruc, D., 2013, State of the arts in gold nanoparticle synthesis, Coord. Chem. Rev., 257 (3-4), 638-665.

[11] Lu, Y.C., and Chou, K.S., 2008, A simple and effective route for the synthesis nano-silver colloidal dispersions, J. Chin. Inst. Chem. Eng., 39 (6), 673-678.

[12] Nguyen, T.H.D., Zhang, Z., Mustapha, A., Li, H., and Lin, M., 2014, Use of graphene and gold nanorods as substrates for the detection of pesticides by surface enhanced Raman spectroscopy, J. Agric. Food Chem., 62 (43), 10445-10451.

[13] Wu, S., Lan, X., Zhao, W., Li, Y., Zhang, L., Wang, H., Han, M., and Tao, S., 2011, Controlled immobilization of acetylcholinesterase on improved hydrophobic gold nanoparticle/Prussian blue modified surface for ultra-trace organophosphate pesticide detection, Biosens. Bioelectron., 27 (1), 82-87.

[14] Aslam, M., Fu, L., Su, M., Vijayamohanan, K., and Dravid, P.V., 2004, Novel one step synthesis of amine-stabilized aqueous colloidal gold nanoparticles, J. Mater. Chem., 14 (12), 1795-1797.

[15] Gusrizal, G., Santosa, S.J., Kunarti, E.S., and Rusdiarso, B., 2016, Dual function of $p$ hydroxybenzoic acid as reducing agent and capping agent in rapid and simple formation of stable silver nanoparticles, Int. J. ChemTech Res., 9 (9), 472-482.

[16] Aji, A., Kunarti, E.S., and Santosa, S.J., 2019, Synthesis of gold nanoparticles using $p$-aminobenzoic acid and $p$-aminosalicylic acid reducing agent, Indones. J. Chem., 19 (1), 68-77.

[17] Nita, R., Trammell, S.A., Ellis, G.A., Moore, M.H., Soto, C.M., Leary, D.H., Fontana, J., Talebzadeh, S.F., and Knight, D.A., 2016, Kinetic analysis of the hydrolysis of methyl parathion using citrate- stabilized $10 \mathrm{~nm}$ gold nanoparticles, Chemosphere, 144, 1916-1919.

[18] Amendola, V., Pilot, R., Frasconi, M., Maragò, O.M., and Latì, M.A., 2017, Surface plasmon resonance in gold nanoparticles: A review, J. Phys.: Condens. Matter, 29 (20), 203002.

[19] Kumari, M., Mishra, A., Pandey, S., Singh, S.P., Chaudhry, V., Mudiam, M.K.R., Shukla, S., Kakkar, P., and Nautiyal, C.S., 2016, Pshyco-chemical condition optimization during biosynthesis lead to development of improved and catalytically efficient gold nanoparticles, Sci. Rep., 6, 27575.

[20] Susanthy, D., Santosa, S.J., and Kunarti, E.S., 2018, The synthesis and stability study of silver nanoparticles prepared using $p$-aminobenzoic acid as reducing and stabilizing agent, Indones. J. Chem., 18 (3), 421-427.

[21] Alvarez-Ros, M.C., Sánchez-Cortéz, S., and GarcíaRamos, J.V., 2000, Vibrational study of the salicylate interaction with metallic ion and surfaces, Spectrochim. Acta, Part A, 56 (12), 2471-2477.

[22] Litvin, V.A., and Minaev, B.F., 2014, The size controllable, one step synthesis and characterization of gold nanoparticles protected by synthetic humic substances, Mater. Chem. Phys., 144 (1-2), 168-178.

[23] Nara, S., Tripathi, V., Singh, H., and Shrivastav, G., 2010, Colloidal gold probe based rapid immunochromatographic strip assay for cortisol, Anal. Chim. Acta, 682 (1-2), 66-71.

[24] Tabrizi, A., Ayhan, F., and Ayhan, H., 2009, Gold nanoparticle synthesis and characterization, Hacettepe J. Biol. Chem., 37 (3), 217-226.

[25] Buduru, P., and Reddy, S.R., 2016, Oxamic acid and $p$-aminobenzoic acid functionalized gold nanoparticles as a probe for colorimetric detection of $\mathrm{Fe}^{3+}$ ion, Sens. Actuators, B, 237, 935-943. 